\title{
Principal static wind loads
}

\author{
Nicolas Blaise, Vincent Denoël* \\ Department of Architecture, Geology, Environment and Construction, University of Liège, Liège, Belgium
}

\section{A R T I C L E I N F O}

\section{Article history:}

Received 28 June 2012

Received in revised form

18 December 2012

Accepted 22 December 2012

\section{Keywords:}

Displacement-response correlation

method

Buffeting wind analysis

Envelope diagram

Extreme value

Equivalent static wind loads

Effective static wind loads

Proper orthogonal decomposition

Singular value decomposition

\begin{abstract}
A B S T R A C T
In current wind design practice, static wind loads are usually defined to obtain, by simple static analyses, the extreme values of any structural response that would be formally obtained with a strict dynamic buffeting analysis. The minimum and maximum values that may reach any response define the envelope. Equivalent static wind loads (ESWLs) allow to recover extreme responses in the envelope. As a first objective, this paper formalizes a general method to determine ESWL, in a nodal basis, by extending the concept of load-response correlation, which is only valid in the background range. The general method, the displacement-response correlation (DRC) method, covers the background and resonant contributions of the considered response. As a second objective, the paper addresses the problem of building a set of static wind loads that adequately reconstructs the envelopes of responses. The concept of principal static wind loads (PSWL) is introduced to form a reduced basis of representative loads well-suited for envelope reconstruction. Its optimality is demonstrated both analytically and with a detailed illustrative example.
\end{abstract}

(c) 2013 Published by Elsevier Ltd.

\section{Introduction}

Since 1960s, buffeting wind analysis of large civil structures has been being handled by stochastic dynamic methods (Liepmann, 1952; Davenport, 1962) which cope with the random nature of the wind excitation and the dynamic behavior of the structures. Convenient ways to characterize the probabilistic nature of wind pressures on civil structures are wind tunnel or in situ measurements. Alternatively, a number of stochastic models for wind turbulence and admittances are available, see Dyrbye and Hansen (1997), Von Karman (1958), Solari and Piccardo (2001). Computational fluid dynamics (Anderson et al., 2009) is another method yet that provides both velocity and pressure fields. On the practical side, structural engineers are used to work with static loads, a reason why the complex probabilistic and time-space-dependent representation of the wind loads mentioned above are commonly replaced by static wind loads.

The use of such a static loading also recasts the design procedure into the well-known format of codes and standards (Standards Australia, 2002; Eurocode 1, 2005; International Standards Organization, 2009) and allows combination with other codified static loads such as snow or self-weight.

\footnotetext{
* Corresponding author. Tel.: +32 436629 30; fax: + 3243669192 .

E-mail addresses: N.Blaise@ulg.ac.be (N. Blaise), V.Denoel@ulg.ac.be (V. Denoël).
}

A way to develop static wind loads for these practical or codification purposes consists in determining equivalent static wind loads (ESWL) such that the application of this equivalent static loading provides the same extreme value of a considered structural response - such as an internal force - as what would result from the dynamic buffeting analysis. Davenport (1967) suggested the first method to determine an equivalent static wind load, although not really termed as such, as the mean wind load amplified by dynamic gust factors, determined specifically for each response. An apparent limitation of this method obviously concerns zero-mean responses.

Holmes (1988) introduced the notion of peak-load pressure distributions and the fact that these distributions are specific for each structural response. With the load-response correlation (LRC) method, Kasperski (1992) established ESWLs, in a Gaussian framework, and defined them as the most probable load profiles corresponding to specific structural responses. These ESWLs are however limited to structures with a background response only. Actually, the ISO-document (International Standards Organization, 2009) has incorporated the LRC method (Kasperski, 2009) and recommends its use to set up design loads. For structures featuring a purely resonant response, ESWLs were expressed as combinations of modal inertial loads. The concept was sketched by Davenport (1985) and, among others, further developed by Holmes (1996) and Zhou et al. (1999). In case of an intermediate structural response between background and resonant, Davenport (1995) and Holmes (1996) underline the interest of using ESWLS as a combination of the two 
limiting cases. The concept was formalized by Chen and Kareem (2001) who use a weighted combination of background and resonant loadings in a hybrid structural analysis, i.e. with a nodal basis analysis for the background component and a modal basis analysis for the resonant component.

The first contribution of this paper concerns the formulation of a method, termed the displacement-response correlation (DRC) method, that computes ESWL in the nodal basis, i.e. without any necessary recourse to the modal basis even if the structure has a resonant behavior. The resulting ESWL is obtained with similar concept of correlation as the LRC method but without the limitation of background structural response. This method is developed in Section 3.

Formally the dynamic analysis of a structure subjected to a probabilistic loading somehow provides minimum and maximum values of the numerous structural responses which are required for the design of the structure. Their minimum and maximum values define the envelope. The design of a structure by means of static wind loads is thus nothing but an envelope reconstruction problem, i.e. the determination of a set of static loadings together with their static responses, such that their envelope matches as best as possible the actual envelope resulting from a more advanced dynamic analysis.

A first basic approach consists in computing the ESWLs associated with every structural responses. For a large and complex structure, the resulting set of loadings is huge but it ensures that the entire envelope is covered. The size of this set is expected to be prohibitive, as the application of an ESWL related to a certain structural response may reconstruct a certain fraction of the actual envelope, with an acceptable tolerance.

It appears thus that a more suitable approach could be to identify, for instance from influence surfaces and some engineering judgement, some representative structural responses along with their corresponding ESWLs. However consideration of only few ESWLs may lead to important underestimation of the envelope, especially if representative structural responses are not selected with care, which is not simple for large structures. Repetto and Solari (2004) have proposed a global loading technique in order to provide global static wind loads that are not associated with a unique structural response by imposing that several selected responses reach their envelope values. The method is however limited to cantilever vertical structures. The same idea was pushed forward by Fiore and Monaco (2009) who evaluate static wind loads as a combination of eigenmodes calculated by spectral proper transformation (Carassale et al., 2001) of fluctuating wind pressures. Recently, Katsumura et al. (2007) have developed a universal static wind load which aims at reproducing simultaneously the absolute maximum responses in all structural members. The universal ESWL is computed as a combination of eigenmodes calculated by covariance proper transformation (CPT) (Best and Holmes, 1983; Bienkiewicz et al., 1995; Solari et al., 2007) of fluctuating wind pressures. The advantage of that method is to have a unique loading, rather than several ESWLs for some chosen responses. Although the concept is appealing, it has been criticized (Li et al., 2009; Zhou et al., 2011; Kasperski, 2009), because it leads to severe underestimations and overestimations of the envelope. Li et al. (2009) have improved the universal static wind load by dividing the responses into several groups based on their influence surfaces. The main disadvantage is that a selection of relevant responses has to be made by engineering judgement and only partially solves the aforementioned drawbacks. Zhou et al. (2011) solve the envelope reconstruction problem with a constrained least-square optimization, with the reconstructed envelope expressed as the structural response to linear combinations of equivalent static load distributions. Ranges of variation of the combination coefficients are obtained by "trial and error" [sic, Zhou et al. (2011)] and the method globally relies on engineering judgement too, which makes it hardly applicable to large structures.

As a second contribution of this paper, we introduce a novel method for the establishment of an optimum set of loadings that globally reproduces the envelope. These loadings are termed principal static wind loads, because they are determined by singular value decomposition. The way the principal static wind loads are defined makes them naturally suitable to solve the envelope reconstruction problem. The concept is introduced in Section 4 and illustrated with a simple example in Section 5.

\section{Statement of the problem}

\subsection{Generalities}

We consider a stationary Gaussian random loading $\mathbf{p}_{\text {tot }}(t)$, representing wind actions, although the concepts could be generalized to other loadings. For convenience the loading is split into a mean part $\boldsymbol{\mu}_{p}$ and a fluctuating part $\mathbf{p}(t)$

$\mathbf{p}_{t o t}=\boldsymbol{\mu}_{p}+\mathbf{p}$.

The dynamic motion $\mathbf{x}(t)$ of a linear structure loaded by this random excitation, in the nodal basis, is obtained by solving the equation of motion

$\mathbf{M} \ddot{\mathbf{x}}+\mathbf{C} \dot{\mathbf{x}}+\mathbf{K x}=\mathbf{p}$,

where $\mathbf{M}, \mathbf{C}$ and $\mathbf{K}$ are $n \times n$ mass, damping and stiffness matrices, respectively.

From a conceptual viewpoint, the right-hand side in (2) is a random process and could be represented as: a complete probabilistic description, a set of synthesized times series, or simply a materialized pressure field in an experimental context. Depending on the kind of representation of $\mathbf{p}$, one or another analysis method may be applied: a stochastic analysis, a sequence of deterministic analyses followed by some statistical treatment or the measurement of the mechanical response.

In any case, the mean nodal displacements $\boldsymbol{\mu}_{x}$ and, secondarily, the background contribution $\mathbf{x}^{(\mathrm{B})}(t)$ may be determined by

$\boldsymbol{\mu}_{x}=\mathbf{K}^{-1} \boldsymbol{\mu}_{p}, \quad \mathbf{x}^{(\mathrm{B})}=\mathbf{K}^{-1} \mathbf{p}$

and the total motion of the structure $\mathbf{x}_{t o t}(t)$ is obtained by

$\mathbf{x}_{\text {tot }}=\boldsymbol{\mu}_{x}+\mathbf{X}$.

Some design quantities such as internal forces, reactions or stresses, referred to as structural responses and denoted by $\mathbf{r}$ are obtained by linear combinations of $\mathbf{x}_{t o t}(t)$

$\mathbf{r}_{t o t}=\mathbf{O} \mathbf{x}_{t o t}$,

where $\mathbf{r}_{\text {tot }}(t)$ is the $m \times 1$ vector of structural responses and $\mathbf{O}$ is an $m \times n$ matrix of influence coefficients. Notice that if the purpose is the design of an entire large structure, $m$ can be quite large and even larger than $n$, the total number of degrees-offreedom (DOF) of the structure. For simplicity, responses are again split into mean $\boldsymbol{\mu}_{r}$ and fluctuating $\mathbf{r}(t)$ contributions

$\mathbf{r}_{t o t}=\boldsymbol{\mu}_{r}+\mathbf{r}$.

Depending on the selected analysis method, deterministic values, minimum $\mathbf{r}^{\min }$ and maximum $\mathbf{r}^{\max }$, of structural responses $\mathbf{r}$ are established, that correspond to the envelope $\left(\mathbf{r}^{\min }, \mathbf{r}^{\max }\right)$. Ultimately, the design of the structural members is based on the design envelope $\left(\mathbf{r}_{\text {tot }}^{\min }, \mathbf{r}_{\text {tot }}^{\max }\right)$ obtained by

$\mathbf{r}_{\text {tot }}^{\min }=\boldsymbol{\mu}_{r}+\mathbf{r}^{\min }, \quad \mathbf{r}_{\text {tot }}^{\max }=\boldsymbol{\mu}_{r}+\mathbf{r}^{\max }$. 


\subsection{Stochastic analysis for the determination of the envelope}

In this section we clarify the establishment of the envelope $\left(\mathbf{r}^{\min }, \mathbf{r}^{\max }\right)$ in the context of a stochastic analysis. We thus assume wind loads are given by a complete probabilistic model.

In a second order analysis in the frequency domain, Eq. (2) is written as

$\mathbf{S}_{x}=\mathbf{H} \mathbf{S}_{p} \overline{\mathbf{H}}^{\mathrm{T}}$,

where $\mathbf{H}(\omega)=\left(-\mathbf{M} \omega^{2}+\hat{\imath} \omega \mathbf{C}+\mathbf{K}\right)^{-1}$ is the nodal transfer function with $\hat{\imath}$ as the imaginary unit and the overbar denotes the complex conjugate operator. Symbols $\mathbf{S}_{x}(\omega)$ and $\mathbf{S}_{p}(\omega)$ represent the power spectral density (PSD) matrices of nodal displacements and external forces, respectively. Integration along circular frequencies of (8) gives the covariance matrix of nodal displacements

$\mathbf{C}_{x}=\int_{-\infty}^{+\infty} \mathbf{H} \mathbf{S}_{p} \overline{\mathbf{H}}^{\mathrm{T}} d \omega$.

Secondarily, the covariance matrix of the background component $\mathbf{x}^{(\mathrm{B})}$ is given by

$\mathbf{C}_{x}^{(\mathrm{B})}=\mathbf{K}^{-1} \mathbf{C}_{p} \overline{\mathbf{K}}^{-\mathrm{T}}=\mathbf{K}^{-1}\left(\int_{-\infty}^{+\infty} \mathbf{S}_{p} d \omega\right) \overline{\mathbf{K}}^{-\mathrm{T}}$,

where $\mathbf{C}_{p}$ is the covariance matrix of the fluctuating part of wind actions.

The deterministic design values $\mathbf{r}^{\min }$ and $\mathbf{r}^{\max }$ correspond to expected values of extrema on a given observation window, e.g. see Rice (1945). Although the formal application of those concepts would yield a different peak factor for each structural response, we consider that

$\mathbf{r}^{\min }=-g \boldsymbol{\sigma}_{r}, \quad \mathbf{r}^{\max }=g \boldsymbol{\sigma}_{r}$

with the assumption that $g$ is a unique peak factor taken equal to 3.5 , for the sake of simplicity, which is anyway the solution adopted in Eurocode 1 (2005), and $\boldsymbol{\sigma}_{r}$ is an $m \times 1$ vector with the standard deviations of the structural responses collected from the main diagonal of the associated covariance matrix

$\mathbf{C}_{r}=\mathbf{O C}_{x} \mathbf{O}^{\mathrm{T}}$.

Eq. (11) defines the envelope of the fluctuating response. The assumption of Gaussian responses leads to a symmetric envelope, see (11). At the opposite, the design envelope is not symmetric, i.e. $\mathbf{r}_{\text {tot }}^{\min } \neq-\mathbf{r}_{\text {tot }}^{\max }$, see (7), except for zero-mean responses.

\subsection{Envelope reconstruction using static wind loads}

In practice, engineers are used to design with a set of static wind loads $\mathbf{p}_{i}^{s}$, with $i \in[1 ; d], d$ being the number of static wind loads considered for the design. These loadings are expected to reproduce sequentially, by static analyses, the envelope $\left(\mathbf{r}^{\min }, \mathbf{r}^{\text {max }}\right)$ obtained with a more advanced analysis, as described in the previous section. With each static loading $\mathbf{p}_{i}^{s}$ is associated a vector of nodal displacements $\mathbf{K}^{-1} \mathbf{p}_{i}^{s}$ and structural responses

$\mathbf{r}_{i}^{s}=\mathbf{O} \mathbf{K}^{-1} \mathbf{p}_{i}^{s}=\mathbf{A} \mathbf{p}_{i}^{s}$,

where $\mathbf{A}=\mathbf{O K}^{-1}$.

The sequential reconstruction of the envelope $\left(\tilde{\mathbf{r}}_{k}^{\text {s,min }}, \tilde{\mathbf{r}}_{k}^{\text {s, } m a x}\right)$ after considering $k$ static wind loads is expressed by the recursive relations

$\tilde{\mathbf{r}}_{k}^{s, \min }=\min \left(\tilde{\mathbf{r}}_{(k-1)}^{s, \min } ;-\mathbf{r}_{i}^{s} ; \mathbf{r}_{i}^{s} ; \mathbf{0}\right), \quad \tilde{\mathbf{r}}_{k}^{s, \max }=\max \left(\tilde{\mathbf{r}}_{(k-1)}^{s, \max } ;-\mathbf{r}_{i}^{s} ; \mathbf{r}_{i}^{s} ; \mathbf{0}\right)$.

Notice that with (14), $2 k$ load cases are associated with the $k$-th reconstructed envelope.
Equivalent static wind loads are a particular set of static loadings that may be used in the envelope reconstruction problem. By analogy with (13), the structural responses under the $i$-th equivalent static wind load reads

$\mathbf{r}_{i}^{e}=\mathbf{O K} \mathbf{K}^{-1} \mathbf{p}_{i}^{e}=\mathbf{A} \mathbf{p}_{i}^{e}$

with the important property now that the envelope value of the $i$-th structural response is well recovered under application of $\mathbf{p}_{i}^{e}$.

Kasperski (1992) defined design static wind loads $\left(\boldsymbol{\mu}_{p}-\mathbf{p}_{i}^{e}\right)$ and $\left(\boldsymbol{\mu}_{p}+\mathbf{p}_{i}^{e}\right)$ including the mean pressure field reproducing the minimum $\mathbf{r}_{\text {tot }, i}^{\max }$ and maximum $\mathbf{r}_{\text {tot, } i}^{\max }$ values of the $i$-th structural response. This formulation of the design loadings is appropriate because the ESWL obviously has to be determined on the fluctuating part of the response only, i.e. not including the average, since first and second order statistics typically exhibit significantly different patterns. The static analysis of the structure under the two design wind loads provides two design static responses $\left(\boldsymbol{\mu}_{r}-\mathbf{r}_{i}^{e}\right)$ and $\left(\boldsymbol{\mu}_{r}+\mathbf{r}_{i}^{e}\right)$ which might be used for the structural design. Notice however that some other methods, proposed by Katsumura et al. (2007) and Zhou et al. (2011), focus on the reconstruction of the maximum absolute value of the envelope by means of static wind loads. These methods encounter some difficulties as the cost function may exhibit some discontinuities, in particular with the least-square approach followed by Zhou et al. (2011).

Although ESWLs are optimum to recover one specific value of the envelope, they are clearly not optimum in the reconstruction of the complete envelope. The objective of this paper is to provide the optimum set of static loadings $\mathbf{p}_{i}^{s}$, with $i \in[1 ; d]$, so as to provide the fastest convergence of the reconstructed envelope $\left(\tilde{\mathbf{r}}_{k}^{s, \min }, \tilde{\mathbf{r}}_{k}^{s, \max }\right)$ towards the actual one $\left(\mathbf{r}^{\min }, \mathbf{r}^{\max }\right)$.

\section{Displacement-response correlation method}

The DRC method aims at producing an ESWL $\mathbf{p}_{i}^{e}$ for the $i$-th structural response $\mathbf{r}_{i}$. The static analysis under $\mathbf{p}_{i}^{e}$ provides the same maximum response $\mathbf{r}_{i}^{\max }$ as what would be obtained with a dynamic buffeting analysis.

In a stochastic dynamic analysis, the covariance matrix between responses and nodal displacements is expressed as

$\mathbf{C}_{r x}=\mathbf{O C}_{x}$,

where $\mathbf{C}_{r x}$ is an $m \times n$ displacement-response covariance matrix. In a Gaussian framework, the conditional probability density function of the displacement $x_{k}$ for given $r_{i}$ is

$p_{x_{k} / r_{i}}=\frac{1}{\sqrt{2 \pi} \sqrt{\left(1-\rho_{x_{k} r_{i}}^{2}\right)} \sigma_{x_{k}}} e^{-\left(x_{k}-r_{i} \rho_{x_{k} r_{i}} \sigma_{x_{k}} / \sigma_{r_{i}}\right)^{2} / 2\left(\sqrt{\left(1-\rho_{x_{k} r_{i}}^{2}\right)} \sigma_{x_{k}}\right)^{2}}$,

where $\rho_{x_{k} r_{i}}$, obtained from $\mathbf{C}_{r x}$, is the correlation coefficient between the nodal displacement $x_{k}$ and response $r_{i}$. Symbols $\sigma_{x_{k}}$ and $\sigma_{r_{i}}$ represent the standard deviations of the nodal displacement and structural response, respectively. In fact, the conditional probability density function (17) is the probability density function of a new Gaussian random variable with a mean value equal to

$\mu_{x_{k} / r_{i}}=r_{i} \rho_{x_{k}} r_{i} \frac{\sigma_{x_{k}}}{\sigma_{r_{i}}}$

which thus corresponds to the most probable displacement $x_{k}$ associated with a given response $r_{i}$. The conditional expectation of the nodal displacement of the $k$-th degree-of-freedom concomitant with the maximum value of the $i$-th response $\mathbf{r}_{i}^{\max }=g \sigma_{r, i}$ is thus obtained by

$\mu_{x_{k} / r_{i}^{\max }}=g \rho_{x_{k} r_{i}} \sigma_{x_{k}}$. 
Therefore the $i$-th equivalent static wind loading associated with the $i$-th structural response $r_{i}$ is defined as

$\mathbf{p}_{i}^{e}=\mathbf{K} \boldsymbol{\mu}_{i}$,

where $\boldsymbol{\mu}_{i}$ is an $n \times 1$ vector whose elements are given by (19).

A major difference, between the equivalent static wind load $\mathbf{p}_{i}^{e}$ and the one resulting from application of the LRC method, is that our ESWL is not necessarily related to an instantaneous load distribution (i.e. a snapshot, or any materialization of it). This actually results from the consideration of viscous and inertial forces in the developments, as shown by (9) and (16). Nevertheless, if the structure has a dominant background or resonant behavior, the ESWL provided by the current DRC method regularly tends towards those obtained with the LRC method, or towards combinations of the modal inertial loads as formalized by Holmes (1996), respectively. Furthermore if the structure has an intermediate background-resonant behavior and can be accurately solved in a reduced modal basis for the resonant contribution, ESWLs obtained with the DRC method virtually correspond to those obtained with the method proposed by Chen and Kareem (2001). In fact, that method provides accurate results with very few computational costs provided an accurate truncation of the modal basis could be a priori formulated. This is not necessarily simple, especially for large and complex structures, should the design also concern secondary elements which could exhibit vibrations in higher modes. With the DRC method, the computational costs are heavier but the question of modal truncation does not arise anymore.

Finally, it should also be noticed that the LRC method provides equivalent loads only where wind forces take place whilst the DRC method can provide loads at any degree-of-freedom of the structure as a result of inertial forces.

ESWLs are defined for each response and are gathered, for the needs of the following developments, in an $m \times n$ matrix $\mathbf{P}^{e}$. This matrix could be large but does not require an excessive computational effort as it simply results from the algebraic transformation (20). This set of equivalent static wind loads is mapped onto a set of structural responses defined as

$\mathbf{R}^{e}=\mathbf{A} \mathbf{P}^{e}$,

where $\mathbf{R}^{e}$ is an $m \times m$ matrix. Notice that

$-g \sigma_{r_{j}}=r_{j}^{m i n} \leq R_{j i}^{e} \leq r_{j}^{\max }=g \sigma_{r_{j}} \quad \forall j \in[1, m], \quad \forall i$,

in words, that application of the $i$-th equivalent static wind load does not result in an overestimation of the envelope anywhere. In a Gaussian framework, this is demonstrated with the complementary condition

$\left|R_{i i}^{e}\right| \geq\left|R_{i j}^{e}\right| \quad \forall i \in[1, m]$.

Indeed, the average of the conditional probability density function of the structural response $r_{j}$, given that the $i$-th structural response reaches its maximum value, is given by

$\mu_{r_{j} / r_{i}^{\max }}=g \rho_{r_{j} r_{i}} \sigma_{r_{j}}$

and it is thus clear that

$-g \sigma_{r_{j}}=r_{j}^{\min } \leq \mu_{r_{j} / r_{i}^{\max }} \leq r_{j}^{\max }=g \sigma_{r_{j}}$.

As a consequence the $j$-th structural response also reaches its maximum or minimum value only if it is correlated with a unit coefficient, in absolute value $\left(\rho_{r_{i} r_{i}}= \pm 1\right)$, with the $i$-th structural response. This indicates that the maximum dynamic responses $\mathbf{r}^{\max }$ lie on the diagonal of $\mathbf{R}^{e}$, at least.

\section{Principal static wind loads}

We define the basis of principal static wind loads (PSWLs) as the principal matrix $\mathbf{P}^{p}$ resulting from the singular value decomposition of $\mathbf{P}^{e}$

$\mathbf{P}^{e}=\mathbf{P}^{p} \mathbf{S} \mathbf{V}^{\prime}$.

The matrix $\mathbf{S}$ has on its main diagonal the principal coordinates and matrix $\mathbf{V}$ collects the combination coefficients to reconstruct the ESWLs. Inspired by common applications of the proper orthogonal decomposition, the principal coordinates in $\mathbf{S}$ are arranged with decreasing magnitude so that only the first few modes may be kept for a sufficiently accurate representation of $\mathbf{P}^{e}$. A first main advantage is that the selection is straightforward since they are ordered by decreasing importance. Moreover, each loading mode (principal loading) is no longer associated with a specific structural response, but rather aims at a global reconstruction of the set of equivalent static wind loads and, as a corollary, of the envelope of structural responses.

The $j$-th principal loading $\mathbf{p}_{j}^{p}$ is normalized such that the corresponding static response $\mathbf{r}_{j}^{p}$ defined as

$\mathbf{r}_{j}^{p}=\mathbf{A} \mathbf{p}_{j}^{p}, \quad \mathbf{R}^{p}=\mathbf{A} \mathbf{P}^{p}$

is somewhere tangent to the envelope. Mathematically

$r_{i}^{\min } \leq R_{i j}^{p} \leq r_{i}^{\max } \quad \forall i \in[1, m], \quad \forall j \in[1, m]$

and

$\forall j, \exists i \in[1, m]: R_{i j}^{p}=r_{i}^{\max } \quad$ or $\quad R_{i j}^{p}=r_{i}^{\min }$.

Consideration of the PSWL offers a global reconstruction of the envelope. In a truncated basis, i.e. if only the first few PSWLs are considered, combinations of the principal loadings could provide a faster convergence to the envelope.

Notice also that, the principal loadings are well-suited for combinations because they are orthogonal vectors due to the SVD operation. The determination of these combination coefficients in the context of the envelop reconstruction problem is addressed in Blaise et al. (2012), while this paper just aims at the introduction of the principal basis and the optimality of the linear subspace they generate.

\section{Illustrations}

\subsection{Structure and dynamic analysis}

A four span bridge is analyzed under wind actions. Each span has a length of $100 \mathrm{~m}$. The finite element model is an assembly of classical 2-D beam elements with two DOFs per node (rotation and vertical displacement). Each span is uniformly divided into 30 finite elements in order to obtain a fine representation of the profiles of internal forces. The number of DOFs is thus equal to 242. Table 1 gives the characteristics of the deck and Fig. 1 depicts the vertical displacements of the first six modes.

A one-dimensional Gaussian velocity field with a mean velocity $U$ equal to $30 \mathrm{~m} / \mathrm{s}$ and a turbulence intensity equal to $16 \%$ is considered. The longitudinal turbulent component $u$ of the velocity field is described by the following power spectral density

Table 1

Characteristics of the deck: $B=$ width; $H=$ height; $\Omega$ =section; $I_{y}$ =inertia; $E=$ Young's modulus; $\rho_{d}=$ mass density; $C_{L}=$ lift coefficient

\begin{tabular}{lllllll}
\hline$B$ & $H$ & $\Omega$ & $I_{y}$ & $E$ & $\rho_{d}$ & $C_{L}$ \\
\hline $30 \mathrm{~m}$ & $4 \mathrm{~m}$ & $1 \mathrm{~m}^{2}$ & $10 \mathrm{~m}^{4}$ & $1 \mathrm{e} 9 \mathrm{~N} / \mathrm{m}^{2}$ & $2500 \mathrm{~kg} / \mathrm{m}^{3}$ & -0.15 \\
\hline
\end{tabular}



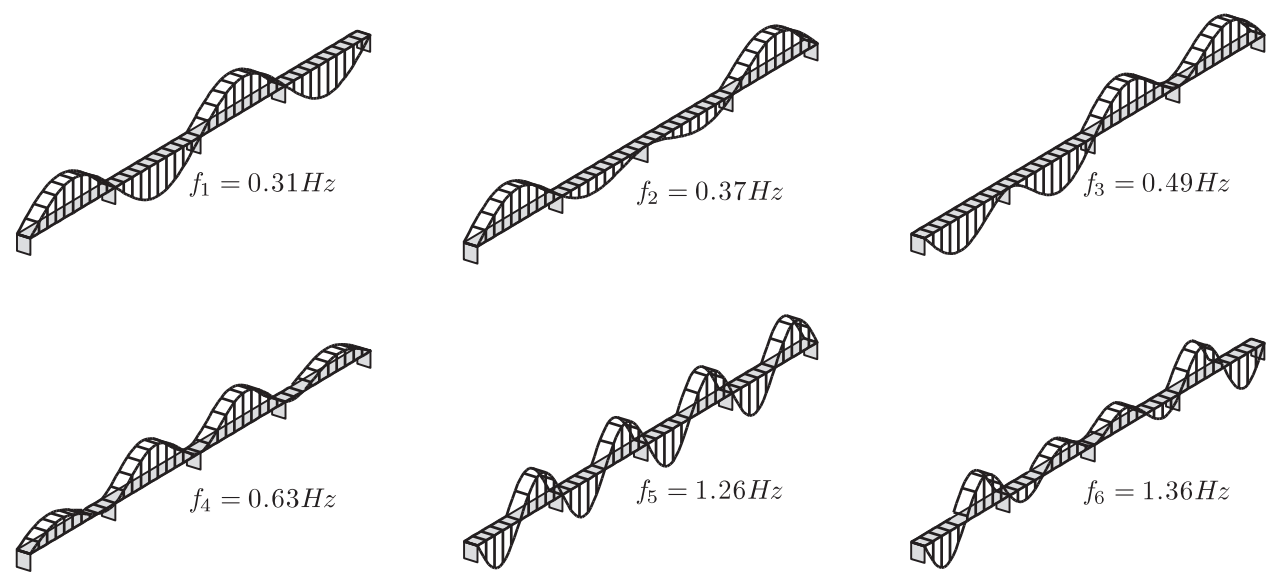

Fig. 1. Modal vertical displacements and associated frequencies.

from Von Karman (1958):

$\frac{\left(\frac{\omega}{2 \pi}\right) S_{u}}{\sigma_{u}^{2}}=\frac{4 f L_{u} / z}{\left[1+70.8\left(f L_{u} / z\right)^{2}\right]^{5 / 6}}$,

where $(\omega / 2 \pi)$ is the frequency, $f=(\omega / 2 \pi) z / U$ is the Monin coordinate, $L_{u}=200 \mathrm{~m}$ is the integral length scale of $u$ and $z=100 \mathrm{~m}$ is the height of the bridge above ground level. The spanwise coherence function of $u$ between two points of the deck separated by a length $\ell$ is modeled by a decreasing exponential (Vickery, 1970)

$\Gamma_{u}=e^{-(2(\omega / 2 \pi) C \ell) / 2 U}$,

where $C$ is the coefficient of coherence taken equal to 8 . For the sake of simplicity, the bridge is analyzed under the lift aerodynamic force and the longitudinal turbulence component only, although from a structural design viewpoint, the vertical component of the turbulence should also be included in the turbulence model, as much as the drag and torque aerodynamic forces should also be considered. The average lift force per unit length, $\mu_{L}$, and average nodal forces, $\boldsymbol{\mu}_{p}$, are respectively given by

$\frac{\mu_{L}}{\frac{1}{2} \rho U B}=C_{L} U, \quad \boldsymbol{\mu}_{p}=\mathbf{F} \mu_{L}$,

where $\mathbf{F}$ is an $n \times 1$ vector that collects the coefficients which transform the lift aerodynamic force per unit length to nodal external forces for each element. This matrix is built up by assuming a suitable interpolation between the nodes of the finite element model, see Denoël and Maquoi (2012). The PSD of the lift aerodynamic force is

$\frac{S_{L}}{\left(\frac{1}{2} \rho U B\right)^{2}}=4 C_{L}^{2} \chi_{L}^{2} S_{u}$,

where $\chi_{L}^{2}$ is the aerodynamic admittance proposed by Davenport (1962)

$\chi_{L}^{2}=\frac{2\left[\frac{7\left(\frac{\omega}{2 \pi}\right)}{U} B-1+\exp \left(-\frac{7\left(\frac{\omega}{2 \pi}\right)}{U} B\right)\right]}{\left(\frac{7\left(\frac{\omega}{2 \pi}\right)}{U} B\right)^{2}}$.
Finally, the PSD matrix of nodal forces is expressed as a function of the PSD of the lift aerodynamic force through

$\mathbf{S}_{p}=\mathbf{F} \boldsymbol{\Gamma} S_{L} \mathbf{F}^{\mathrm{T}}$,

where $\boldsymbol{\Gamma}$ is an $n \times n$ matrix of aerodynamic admittances that takes into account the imperfect correlation of the turbulent component $u$ of the velocity field using (31).

A Rayleigh damping matrix $\mathbf{C}$ is constructed by imposing a damping coefficient $\xi$ in the first and fourth modes. Three damping ratios $0.5 \%, 1.5 \%$ and $4.5 \%$ are used to study a resonant, a background-resonant and a background, behavior of the structure, respectively. In the sequel, it is chosen to focus exclusively on the bending moment but the same developments could be performed with several other types of responses. The number $m$ is thus equal to 121 and the bending moments are numbered from left to right, as for the spans and supports.

Fig. 2(a) shows the bending moments under the uniform average lift. The shape of this diagram is well-known. Fig. 2(b) depicts the maximum dynamic bending moments, obtained as explained in Section 2.2, for the three cases. As expected, maxima occur in side spans at the extremities and the resonant case, $\xi=0.5 \%$, provides the largest maximum fluctuating bending moments. In order to evaluate the relative importance of the background $\sigma_{r}^{2,(\mathrm{~B})}$ and resonant $\left(\sigma_{r}^{2}-\sigma_{r}^{2,(\mathrm{~B})}\right)$ components in the responses, the background-resonant ratio $b=\sigma_{r}^{2,(\mathrm{~B})} /\left(\sigma_{r}^{2}-\sigma_{r}^{2,(\mathrm{~B})}\right)$ for each bending moment is computed and given in Fig. 2(c). These ratios show the same profile for the three cases. Near supports, the background component for each case is more important than in the span which simply results from the smallness of inertial forces near supports.

\subsection{Equivalent static wind loads}

For the case $\xi=1.5 \%$, equivalent static wind loads are computed to recover the envelope for six considered bending moments which are localized by dots in Fig. 2(b) with their associated number. They correspond to the two maximum bending moments in the first two spans, to the bending moment on the second support and to the three local minima in the first and second spans. The corresponding equivalent static wind loads $\mathbf{p}_{i}^{e}$ and responses $\mathbf{r}_{i}^{e}(i \in\{14,27,31,37$, $46,57\})$ are computed with the DRC method, and shown in Fig. 3. Although ESWLs comprise both transverse forces and moments, only transverse forces are represented in Fig. 3; the same convention holds in the following figures. Fig. 3 also illustrates the vertical displacement component of the influence line $\mathbf{a}_{i}$ corresponding to the $i$-th bending moment and the correlation coefficient $\boldsymbol{\rho}_{i}$ between the $i$-th bending moment and the bending moment profile (obtained with the 
a

Bending moments

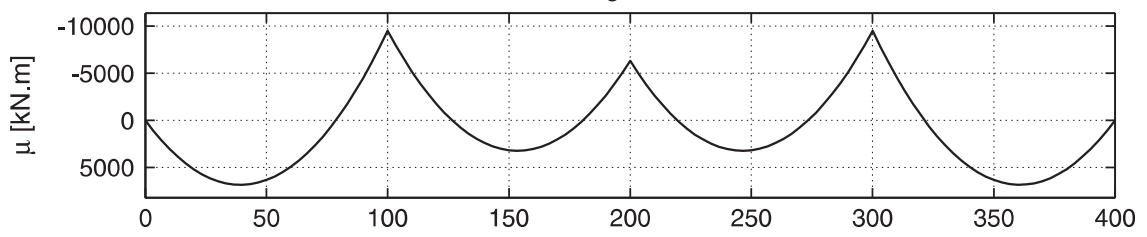

b

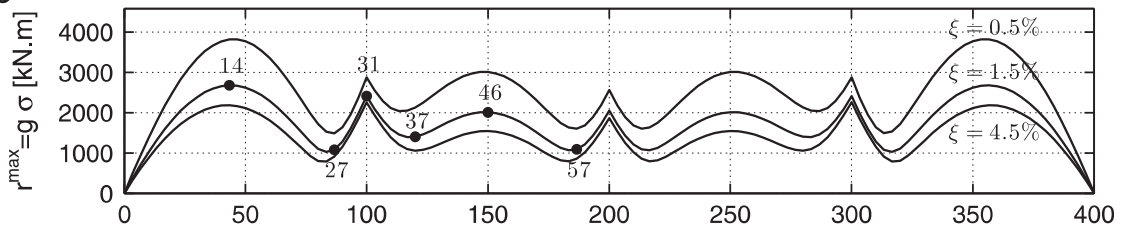

C

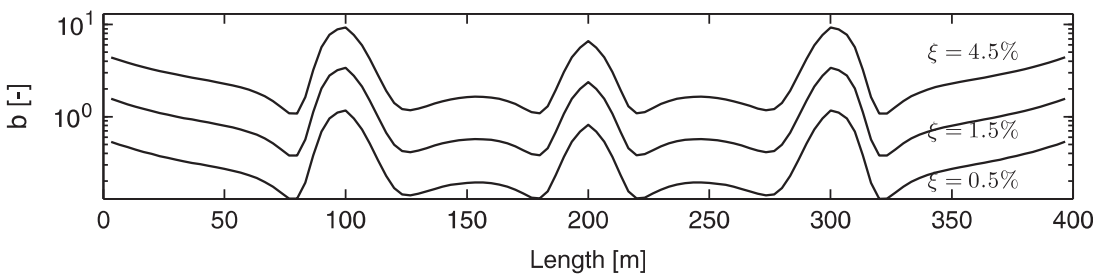

Fig. 2. Constant (a) and (b) maximum bending moments diagrams and (c) background-resonant ratios of the bending moments for the three studied cases.

stochastic dynamic analysis). Fig. 3 also depicts the sequential reconstruction of the envelope $\tilde{\mathbf{r}}_{k}^{e}$ using the responses for the considered bending moments.

As expected, the shapes of the loads are related to the influence lines of the considered responses. Actually, the loadings do not match precisely the influence line due the coherence in the velocity field. It could be observed that the less the coherence there is, the more equivalent static wind loads match the influence lines of the considered responses.

Notice that loads derived with the DRC method virtually correspond to the ones obtained with the method proposed by Chen and Kareem (2001), if the first four modes are considered in the modal basis.

Fig. 3 illustrates that the response $\mathbf{r}_{i}^{e}$ under a specific equivalent static wind load lies within the envelope but also partially reconstructs the envelope at other sections than the considered one, especially when the correlation is close to unity in magnitude, see (25). For example, minimum bending moments in the first span are well reconstructed under the fourth load for which the considered bending moment is strongly negatively correlated with bending moments in the first span.

Although the reconstructed envelope $\tilde{\mathbf{r}}_{k}^{e}(k=1 \ldots 6)$ monotonically converges towards the actual envelope, only bending moments close to the considered sections are well represented after six equivalent loadings. Indeed, in the third and fourth spans, the reconstruction of the envelope is insufficient. It could be improved by considering ESWL related to bending moments at the intermediate and third supports, as well as the maxima and local minima in the third and fourth spans. This would result in a total of 13 bending moments to obtain an acceptable reconstruction of the envelope, globally.

For this simple structure, the selection of the bending moments is accessible but can be grueling for a large one. Moreover, the accuracy of the envelope reconstruction is tributary of the arbitrarily chosen bending moments, which makes the basis of ESWLs inefficient for the envelope reconstruction problem.

\subsection{Principal static wind loads}

Fig. 4 shows the first four principal static wind loads (computed from the 121 ESWLs) and the corresponding responses for the three studied cases. The left column in Fig. 4, collects the static loads and responses for a structure with a dominant resonant behavior $(\xi=0.5 \%)$. These loads are directly related to the vertical component of the modal inertial loads introduced by Chen and Kareem (2001) with slight dissimilarity at supports. In this simple case with uniform mass and bending stiffness, the modal inertial loads are affine to the mode shapes, see Fig. 1.

The right column in Fig. 4 corresponds to a dominant background behavior $(\xi=4.5 \%)$. The same PSWL could be obtained by SVD of the ESWLs computed with the LRC method. The middle column in Fig. 4, represents the basis for an intermediate background-resonant behavior. In that case, the singular value decomposition automatically provides loads with similarities with both limit cases. Also, some loadings in the three cases have close shapes but differ in position because of the behavior of the structure, see for example, the first and second loadings for the cases $\xi=0.5 \%$ and $\xi=1.5 \%$. In general, PSWLs are no longer associated with a specific bending moment but are the principal components, ordered by importance, of all the ESWLs. This is confirmed by their symmetry with respect to the vertical center of the bridge.

Consequently, several extrema are recovered with a single loading. Moreover, the responses ensure a global reconstruction of the envelope.

We now seek to confront the proposed PSWL basis with the basis formed by the CPT modes used for the establishment of the universal loading as suggested by Katsumura et al. (2007). The covariance proper transformation (CPT) is applied to the covariance matrix of external forces

$\left(\mathbf{C}_{p}-\mathbf{C}_{c} \mathbf{I}\right) \mathbf{P}^{c}=0$,

where $\mathbf{C}_{c}$ is a diagonal covariance matrix of principal components ordered by decreasing variances, $\mathbf{I}$ is the identity matrix and $\mathbf{P}^{c}$ collects the CPT loading modes. Fig. 5 shows the CPT loading modes on the upper part and the associated bending moments on the lower part. This time, envelopes of the structural responses are not represented because the CPT loading modes should also be normalized differently for each case.

The only similarity between the CPT loading modes and the principal loadings is that the first CPT modes resemble the first PSWL in case of a background behavior of the structure. The other 


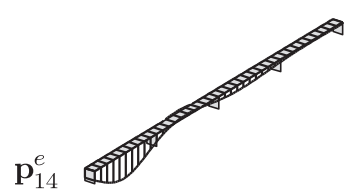

$\mathbf{a}_{14}$
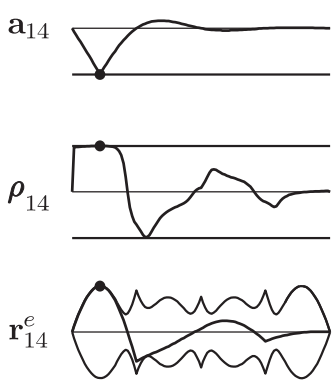

$\tilde{\mathbf{r}}_{1}^{e}$
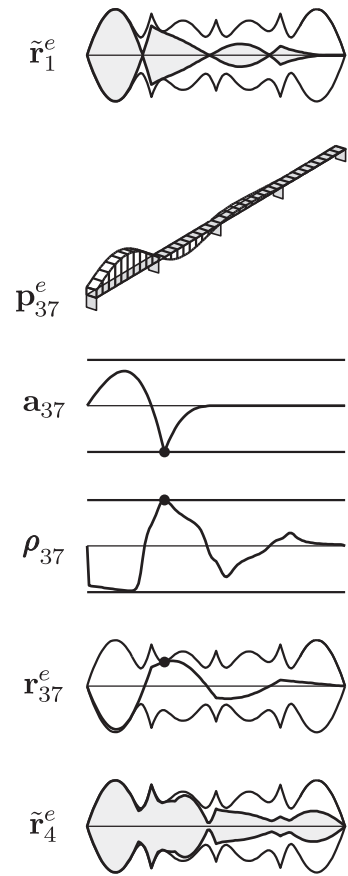
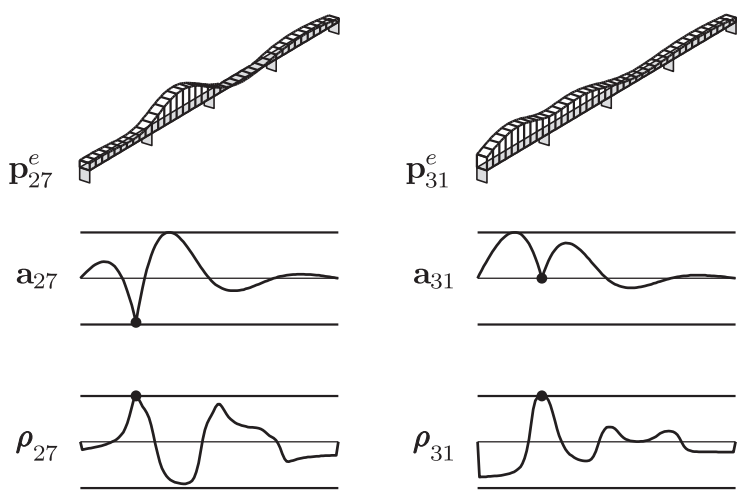

$\mathbf{r}_{27}^{e}$
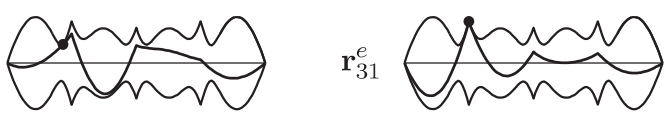

$\tilde{\mathbf{r}}_{2}^{e}$
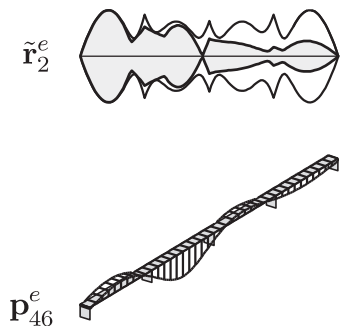

$\mathbf{a}_{46}$

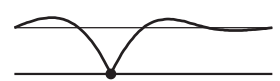

$\rho_{46}$
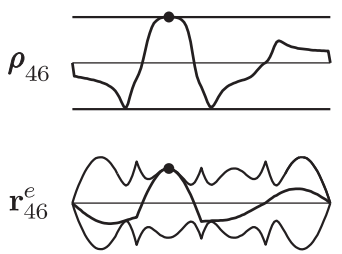

$\tilde{\mathbf{r}}_{5}^{e}$

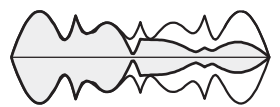

$\tilde{\mathbf{r}}_{3}^{e}$

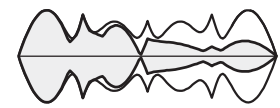

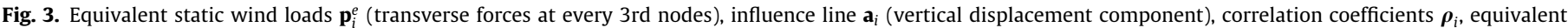
static bending moments $\mathbf{r}_{i}^{e}$ and reconstructed envelope $\tilde{\mathbf{r}}_{k}^{e}$ for the case $\xi=1.5 \%$. Dots identify the considered bending moments.

two CPT modes do not exist in any of the three PSWL bases. The CPT loading modes could thus be adequate in the reconstruction of the structural response only if the structure exhibits a background behavior.

The main disadvantage of the CPT loading modes is that, in contrast with principal static wind loads, they do not take into account the mechanical behavior of the structure and a possible resonant contribution in the response.

The normalized cumulative summation of the principal coordinates $S_{i i}$ for the three cases and for the principal components obtained with CPT $C_{c, i i}$ are shown in Fig. 6. The first four PSWL reproduce more than $90 \%$ of the total sum of the principal coordinates. For both bases, the selection of a minimum number of representative static wind loads is automatic and straightforward because they are ordered by decreasing importance.

Fig. 7 gives the reconstruction $\tilde{\mathbf{r}}_{k}^{\text {p.max }}$ using PSWLs (upper half of each graph) and the reconstruction $\tilde{\mathbf{r}}_{k}^{c, \text { min }}$ (lower half of each graph) using CPT loading modes.
The reconstruction of $\mathbf{r}^{\max }$ with PSWL and $\mathbf{r}^{\min }$ with CPT loading modes is represented only for an easier comparison on the same graph of the envelope reconstruction using the two different methods.

For any behavior of the structure, background, resonant or combined, the first three principal loadings contribute significantly to the reconstruction of the envelope. Actually, after consideration of the first three principal loadings, any additional principal loading just provides a marginal contribution. The same remark holds for CPT loading modes, with four modes instead of three, though.

Principal static wind loads globally perform better than CPT loading modes, especially for the two cases $\xi=0.5 \%$ and $1.5 \%$. For the case $\xi=4.5 \%$, the reconstructed envelope using principal loadings or CPT loading modes is very similar, which illustrates that CPT modes indeed perform well with a background response. In case of resonant response $(\xi=0.5 \%)$, the envelope reconstructed with five CPT loading modes leads to important 


$$
\xi=0.5 \%
$$
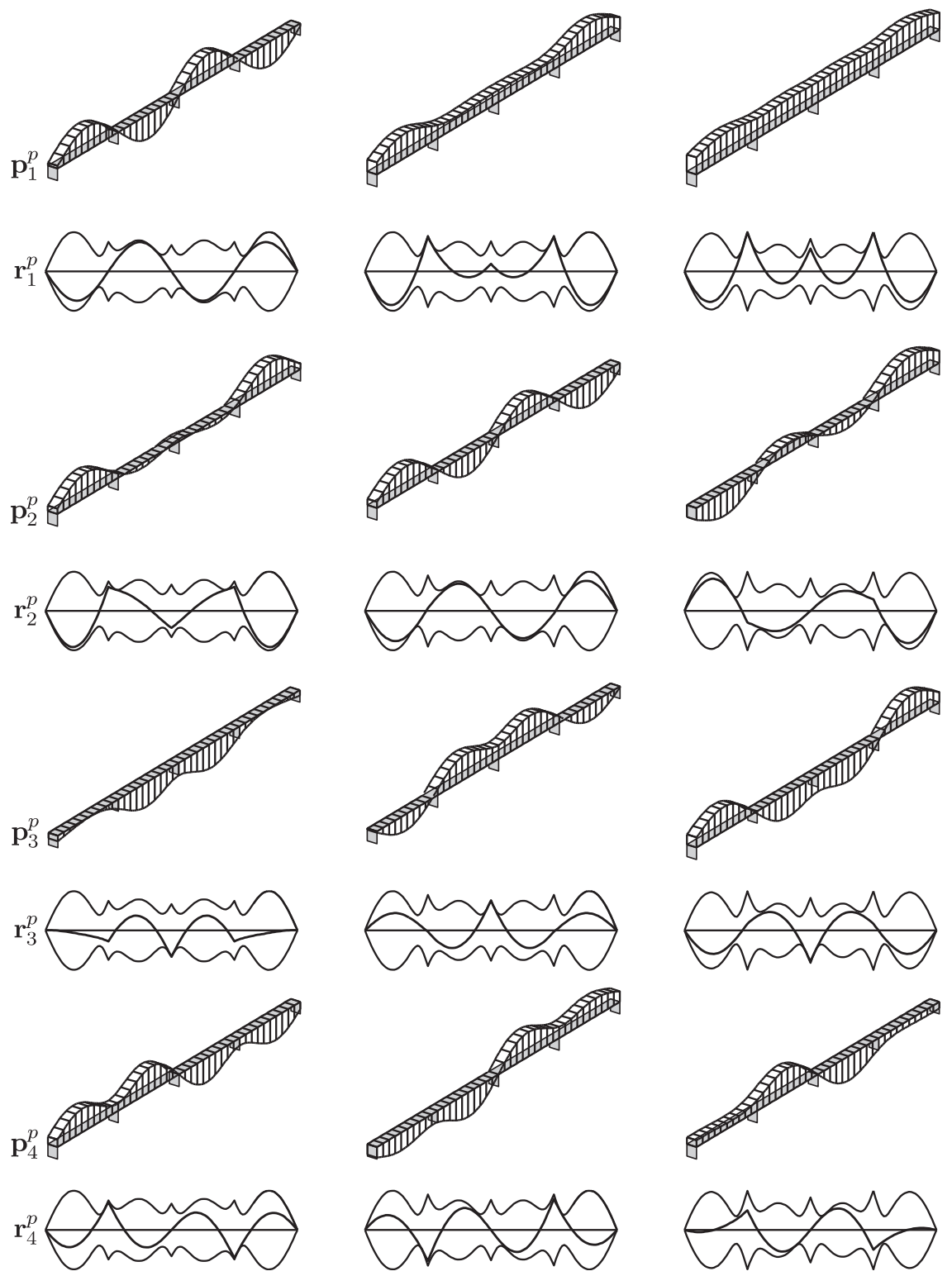

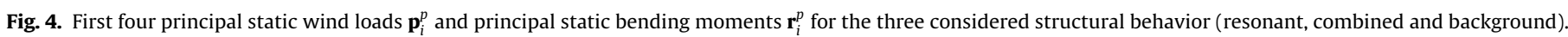
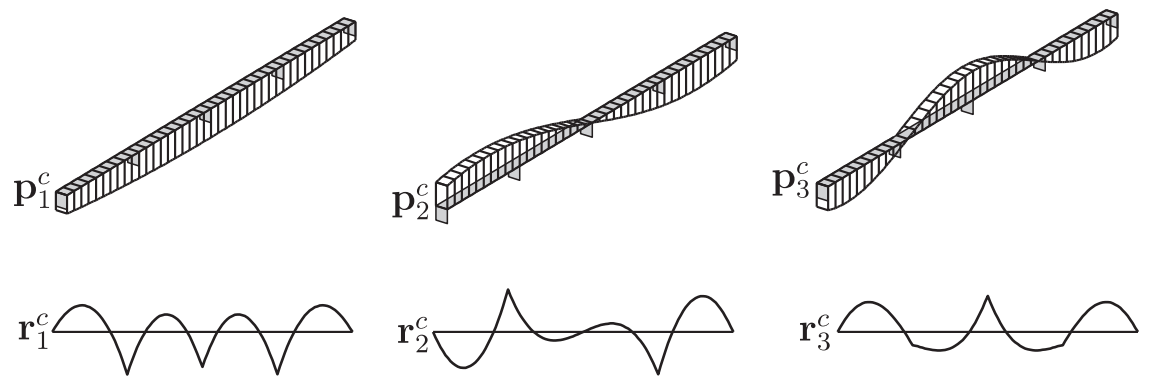

Fig. 5. First three CPT loading modes and static bending moments.

underestimation, especially in side spans and where the bending moment is locally minimum in the second and third spans. These correspond to abscissa where the bending moment is resonant, see Fig. 2(c).

\subsection{Combinations of static wind loads}

In the former section, we have studied the adequacy of the principal loadings, each of them being considered separately, in 
the reconstruction of the envelope. However Eq. (26) suggests that principal loadings $\mathbf{P}^{p}$ are generated in view of possible linear combinations. By essence, linear combinations of the principal loadings allow the reconstruction of the full set of equivalent static wind loads. The objective of this section is to assess the optimality of the subspace spanned by a limited number of static loadings, and not by some static loadings as in the former section.

More precisely, several combinations of the principal static wind loads $\mathbf{p}^{g}$ and the associated structural responses $\mathbf{r}^{g}$ are obtained by

$\mathbf{p}^{g}=\mathbf{P}^{p} \mathbf{q}^{p}, \quad \mathbf{r}^{g}=\mathbf{A} \mathbf{p}^{g}$,

where $\mathbf{q}^{p}$ is a vector of arbitrary combination coefficients. The only limitation on the combination coefficients is that the resulting structural response $\mathbf{r}^{g}$ does not overestimate the actual envelope and that the envelope is reached in at least one particular abscissa (tangency condition). As a consequence, in the multi-dimensional space related to the envelope, $\mathbf{r}^{g}$ is a parametric representation of the subspace spanned by the principal static wind loadings collected in $\mathbf{P}^{p}$.

As an example, the area limited by the curve in Fig. 8(a) in the $\left(q_{1}^{p}, q_{2}^{p}\right)$ space represents the set of combination coefficients $\left(q_{1}^{p}, q_{2}^{p}\right)$ that would result in a structural response $\mathbf{r}^{g}$ lying inside the

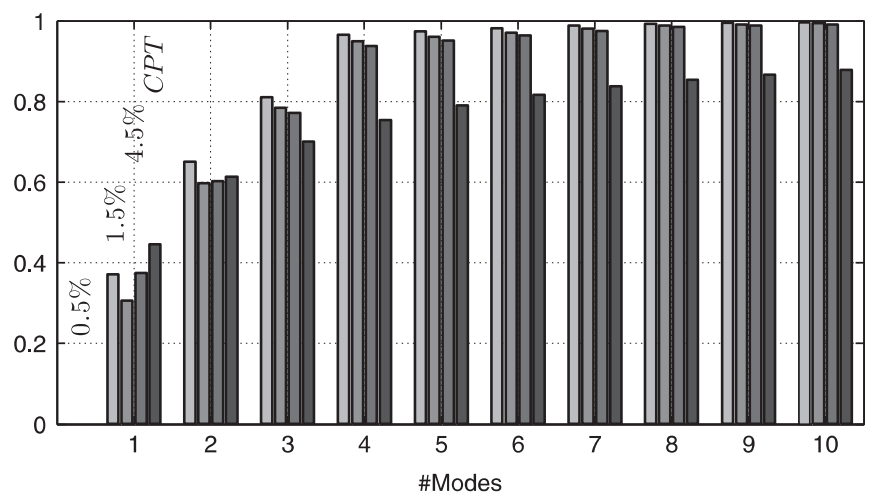

Fig. 6. Normalized cumulative summation of the principal coordinates of the principal loadings and of the CPT loading modes. actual envelope. Conversely, any combination outside that curve results in an overestimation of the actual envelope. The somewhat circular shape of that border indicates that the principal value decomposition has generated an isotropic combination space. The same developments operated with the CPT loading modes reveal a diamond-shaped border, see Fig. 8(b).

From a practical viewpoint, the curves in Fig. 8 are obtained by shooting in a random direction $\left(q_{1}^{p}, q_{2}^{p}\right)$ generated with a Monte Carlo simulation technique, then by scaling the generated combination in order to restore the tangency condition with the actual envelope. The same technique is applied for the combination of the first three principal loading modes and of the first three CPT modes, see Fig. 9(a) and (b), respectively.

Fig. 9 depicts the scaled coefficients for the first three PSWLs for the three cases and for the first three CPT loading modes for the case $\xi=1.5 \%$. Only the case $\xi=1.5 \%$ is shown for the CPT loading modes because the allowed area is almost unchanged. On the contrary, the allowed area for the combination coefficient of the PSWLs adapts as a result of the automatic adaptation of the PSWL basis to the structural behavior. Notice that the allowed subspaces $\left(q_{1}, q_{2}\right),\left(q_{2}, q_{3}\right)$ and $\left(q_{3}, q_{1}\right)$ are represented with thick lines as the equator and two principal meridians.

The envelopes $\tilde{\mathbf{r}}_{k}^{g_{p}, \max }$ and $\tilde{\mathbf{r}}_{k}^{g_{c}, \min }$ reconstructed with the allowed subspace of combination coefficients provide, for the basis of principal loadings and for the basis of CPT modes, the results given in Fig. 10. Combination of two principal loadings offers, with few exceptions, a more accurate reconstructed

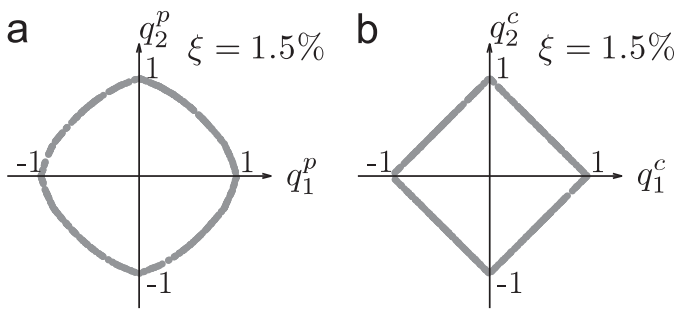

Fig. 8. Scaled combination coefficients for (a) two PSWLs and (b) two CPT loadings modes obtained for $\xi=1.5 \%$.

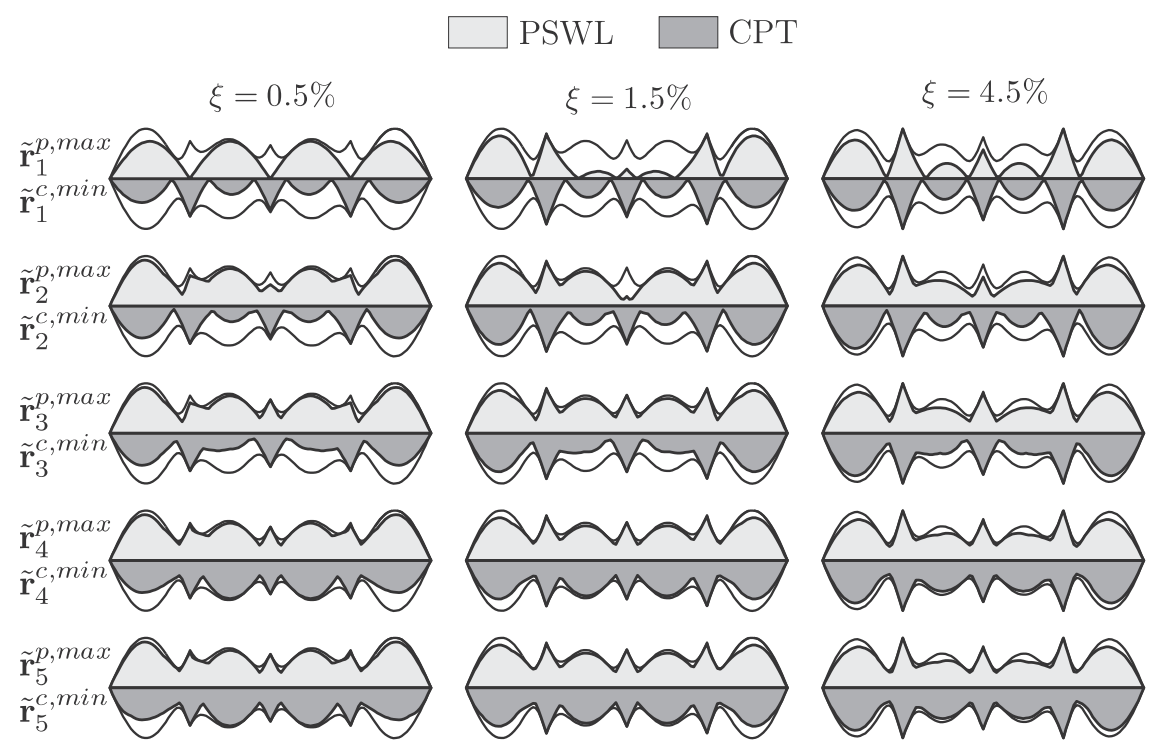

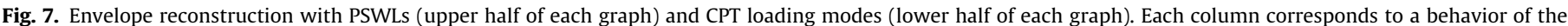
structure associated with a specific damping ratio. 
a

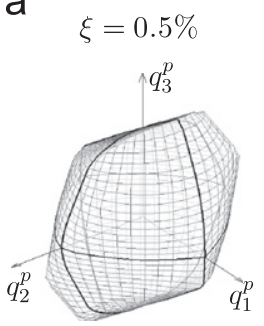

$\xi=1.5 \%$

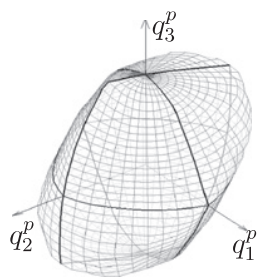

$$
\xi=4.5 \%
$$

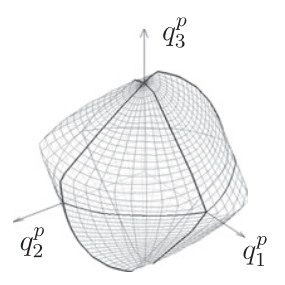

b

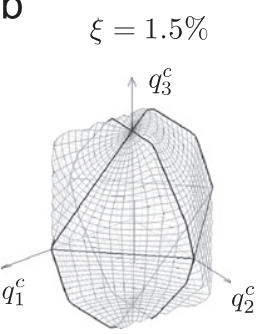

Fig. 9. Scaled combination coefficients for (a) three PSWLs for the three cases and (b) three CPT loadings modes for the case $\xi=1.5 \%$ (on the right).

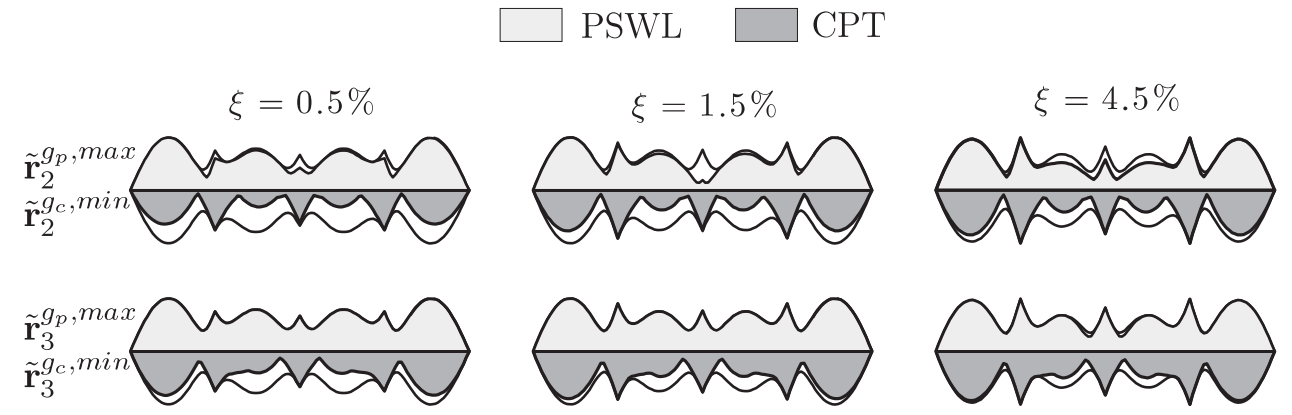

Fig. 10. Envelopes that may be reconstructed by combinations of two or three PSWLs (upper half of each graph) and CPT loading modes (lower half of each graph).

envelope $\tilde{\mathbf{r}}_{2}^{g_{p}, \max }$ than what could be obtained with up to five principal loadings $\tilde{\mathbf{r}}_{k}^{p, \max }(k=1, \ldots, 5)$ considered separately, as in Fig. 7. Moreover, combination of the three principal loadings provides a nearly perfect reproduction of the actual envelope.

On the contrary, the lower half of the plots indicates that two or even three CPT loading modes and any combination of them do not increase significantly the reconstruction of the envelope, in comparison with Fig. 7, especially for the cases $\xi=0.5 \%$ and $\xi=1.5 \%$.

This demonstrates again the optimality of the basis of principal static wind loads in the global reconstruction of the envelope of structural responses. This may be partially attributed to the versatility of the principal loadings with respect to the characteristics of the structural response.

\section{Conclusions}

In structural engineering, static wind loads are used since decades because of their numerous advantages. The static wind loads aim at covering the envelope values of any structural responses. If one response is considered, many methods have been developed in order to build the equivalent static wind load.

In this context, we have extended the concept of loadresponse correlation, confined in the background range, to a dynamic nodal analysis by the displacement-response correlation method.

Moreover, in order to reconstruct the entire envelope in a whole structure with static analyses, a reduced basis of principal loadings has been proposed. These loadings, obtained by singular value decomposition, represent a more global information on the static wind loads that maximize all of the structural responses for any structure.

It has been demonstrated with an illustrative example that the principal static wind loads or combination of them are well suited for an accurate reconstruction of the envelope. This makes the principal static wind loads an optimum basis for the structural design.
The concept is robust in view of its mathematical definition and is applicable to experimental or CFD data, probabilistic description of the loading and codification purposes.

\section{References}

Anderson, J.D., Degroote, J., Degrez, G., Dick, E., Grundmann, R., Vierendeels, J., 2009. Computational Fluid Dynamics. An Introduction, 3rd ed. Springer, Rhode-Saint-Genèse.

Best, R.J., Holmes, J.D., 1983. Use of eigenvalues in the covariance integration method for determination of wind load effects. Journal of Wind Engineering and Industrial Aerodynamics 13, 359-370.

Bienkiewicz, B., Tamura, Y., Ham, H.J., Ueda, H., Hibi, K., 1995. Proper orthogonal decomposition and reconstruction of multichannel roof pressure. Journal of Wind Engineering and Industrial Aerodynamics 54, 369-381.

Blaise, N., Hamra, L., Denoël, V., 2012. Principal static wind loads on a large roof structure. In: Proceedings of the 12th ANIV Conference of Wind Engineering In Vento.

Carassale, L., Piccardo, G., Solari, G., 2001. Double modal transformation and wind engineering applications. Journal of Engineering Mechanics-ASCE 127, 432-439.

Chen, X.Z., Kareem, A., 2001. Equivalent static wind loads for buffeting response of bridges. Journal of Structural Engineering-ASCE 127, 1467-1475.

Davenport, A.G., 1962. The response of slender line-like structures to a gusty wind In: Proceedings of the Institution of Civil Engineers, vol. 23, pp. 389-408.

Davenport, A.G., 1967. Gust loading factors. Proceedings of the American Society of Civil Engineers. Journal of Structural Division, 93, pp. 11-34.

Davenport, A.G., 1985. The representation of the dynamic effects of turbulent wind by equivalent static wind loads. In: Proceedings of the International Engineering Symposium on Structural Steel, pp. 1-13.

Davenport, A.G., 1995. How can we simplify and generalize wind loads. Journal of Wind Engineering and Industrial Aerodynamics 54, 657-669.

Denoël, V., Maquoi, R., 2012. The concept of numerical admittance. Journal of Archives of Applied Mechanics 82 (10), 1337-1354.

Dyrbye, C., Hansen, S.O., 1997. Wind Loads on Structures. Wiley \& Sons, Ltd..

Eurocode 1, 2005. Actions on Structures-General Actions-Part 1-4: Wind Actions.

Fiore, A., Monaco, P., 2009. Pod-based representation of the alongwind equivalent static force for long-span bridges. Wind and Structures, An International Journal 12, 239-257.

Holmes, J.D., 1988. Distribution of peak wind loads on a low-rise building. Journal of Wind Engineering and Industrial Aerodynamics 29, 59-67.

Holmes, J.D., 1996. Along-wind response of lattice towers-III. Effective load distributions. Engineering Structures 18, 489-494.

International Standards Organization, 2009. ISO 4354-Wind Actions on Structures.

Kasperski, M., 1992. Extreme wind load distributions for linear and nonlinear design. Engineering Structures 14, 27-34. 
Kasperski, M., 2009. Incorporation of the lrc-method into codified wind load distributions. In: Proceedings of the 7th Asia-Pacific Conference on Wind Engineering.

Katsumura, A., Tamura, Y., Nakamura, O., 2007. Universal wind load distribution simultaneously reproducing largest load effects in all subject members on large-span cantilevered roof. Journal of Wind Engineering and Industrial Aerodynamics 95, 1145-1165.

Li, Y.Q., Wang, L., Tamura, Y., Shen, Z.Y., 2009. Universal equivalent static wind load estimation for spatial structures based on wind-induced envelope responses. In: Symposium of the International Association for Shell and Spatial Structures (50th. Valencia).

Liepmann, H.W., 1952. On the application of statistical concepts to the buffeting problem. Journal of Aeronautical Sciences 19, 793-800.

Repetto, M.P., Solari, G., 2004. Equivalent static wind actions on vertical structures. Journal of Wind Engineering and Industrial Aerodynamics 92, 335-357.

Rice, S., 1945. Mathematical analysis of random noise. Bell System Technical Journal 24, 45-156.
Solari, G., Carassale, L., Tubino, F., 2007. Proper orthogonal decomposition in wind engineering. Part 1: a state-of-the-art and some prospects. Wind and Structures 10, 153-176.

Solari, G., Piccardo, G., 2001. Probabilistic 3-d turbulence modeling for gust buffeting of structures. Probabilistic Engineering Mechanics 16, 73-86.

Standards Australia, 2002. Structural Design Actions. Part 2: Wind Actions. Australian/New Zealand Standard as/nzs 1170.2:2002.

Vickery, B., 1970. On the reliability of gust loading factors. In: Proceedings of the Technical Meet Concerning Wind Loads on Buildings and Structures, National Bureau of Standards, Washington, pp. 93-104.

Von Karman, T., 1958. Progress in the statistical theory of turbulence. Proceedings of the National Academy of Sciences USA 34, 530-539.

Zhou, X., Gu, M., Li, G., 2011. Application research of constrained least-squares method in computing equivalent static wind loads. In: Proceedings of the 13th International Conference on Wind Engineering.

Zhou, Y., Gu, M., Xiang, H., 1999. Alongwind static equivalent wind loads and responses of tall buildings. Part ii: effects of mode shapes. Journal of Wind Engineering and Industrial Aerodynamics 79, 151-158. 